Volume 11, No.1, January - February 2022

International Journal of Advanced Trends in Computer Science and Engineering

Available Online at http://www.warse.org/IJATCSE/static/pdf/file/ijatcse061112022.pdf

https://doi.org/10.30534/ijatcse/2022/061112022

\title{
Biometric Based Authentication System for Computer Based Assessment
}

\author{
Achimba Terfa ${ }^{1}$, Alaaga James ${ }^{2}$, Kwaghbee Sever ${ }^{3}$, Vanen Samuel ${ }^{4}$ \\ ${ }^{1}$ Department of Mathematics and Computer Science, University of Mkar, Benue State, Nigeria, terfaachimba@gmail.com \\ ${ }^{2}$ National Agency for Food and Drug Administration (NAFDAC), Abuja, Nigeria, jimonline4u@gmail.com \\ ${ }^{3}$ Department of Mathematics and Computer Science, University of Mkar, Benue State, Nigeria, kwaghbees@gmail.com \\ ${ }^{4}$ Department of Mathematics and Computer Science, University of Mkar, Benue State, Nigeria, vanensamuel2004 @ gmail.com
}

Received Date : December 09, 2021 Accepted Date :January 11, 2022 Published Date : February 06, 2022

\begin{abstract}
Electronic-Learning has attracted a lot of attention in recent years. This is evident by the increase in population shift from the traditional way of learning. An important aspect of Electronic Learning is the assessment of students. Since the traditional way of student assessment is characterized by issues such as insecurity, they are replaced by Electronic means of assessment. Most Electronic assessment systems make use of the knowledge based and object based methods for authentication, which can be compromised,shared or transferred, resulting into issues such as impersonation.This research focuses on developing a system that uses facial biometric traits in authenticating candidtates for Electrronic Examination. The research uses some mathematical models based on the Principal Component Analysis(PCA) technique for face recognition. The face is prefered for its ability to offer continous authentication, which will eradicate isues such as impersonation. The system was tested in the department of Mathematics and Computer Science, University of Mkar, Mkar, Nigeria and it offers a reliable platform for authentication.
\end{abstract}

Key words: E-assessment; authentication; Computer Based Assessment; E-learning; examination

\section{INTRODUCTION}

Information and Communication Technologies (ICTs) is growing at a rapid rate and has continued to change people's lifestyle. The use of various ICT tools has impacted the society by creating new ways to access, process and deliver useful knowledge [1]. Information and Communication Technology has improved our lives and provided a new dimension of operation by bringing regular day-to-day tasks to our doorsteps. Web Technology, an aspect of ICT is wide spread in all aspect of life and has changed the mode of communication and information for humans [2-4].

The evolution of Information and Communication Technologies provide an improved and efficient way of delivering contents to remote students. Web based learning activities offer decreased delivery costs, speed in knowledge acquisition, self-paced learning, easy management for large groups of students etc. These activities allow the students to adapt their schedule and attend their preferred courses regardless from where they are offered [5].

Over the years, the population of people that participate in electronic learning has increased in significant dimension, indicating how electronic learning is replacing the conventional (old or traditional) way of learning [6].

One important aspect of electronic-learning is the assessment of students. Assessment for Learning is the process of seeking and interpreting evidence for use by learners and their teachers to decide where the learners are in their learning, where they need to go and how best to get there[7]. The learning capability of students is judged by student assessment which is mostly through examinations. Therefore, the need for examinations today in universities, schools, colleges and even companies for recruitment cannot be overemphasized.

The traditional way of assessment (paper based examinations) has a lot of issues. Also, conducting traditional examinations during pandemic situations is another big challenge for most of the institutions [8].

In an electronic-learning environment, assessment is done through an electronic means known as electronic assessment (e-assessment), which eradicates most of the problems with the traditional form assessment.

Computer-Based Assessment, also known as e-assessment is the use of information technology for any assessment- related activity [3]. E-assessment is an integral and vital component of E-learning. It can improve the standards of student's assessment whereas the conventional way of assessment requires more effort on the part of students and invigilators. E-assessment can be conducted for remote candidates at any time and does not incur higher cost as compared to traditional examinations, does not necessarily involve arrangement of examination centers and doesn't require invigilators [9].

E-examination can be delivered through offline and online modes. The offline mode is delivered without the use of Internet, strictly operated on machine software and authentication is done on single Personal Computer (PC) while the online mode is delivered through an internal network (or the Internet) and its distributed authentication servers to locally provide access to the question banks through server-client interactions among computers in the network [10-11]. E-examination is timely, has online real-time 
capabilities, is flexible in scoring and has diversity in question type or format. It is reliable, impartial, has storage efficiency, enhanced question styles and provides safety of scripts [12]. Despite the numerous advantages offered by E-learning and E-assessment, there are several limitations/challenges. One of the challenges of both traditional and E-assessment is insecurity. Since the inception of e-learning, there have been security and reliability issues and these pose various threats especially when examinations are held electronically.

The implementation of e-assessment in an e-learning environment can only be successful only if trust, privacy and security are secured and strengthened. One way to mitigate security breach during electronic examination is to identify, authenticate and monitor candidates during examinations [2]. Authentication can be defined as a technique used by a computer in verifying the identity of individuals who want to have access to that systems' resources. It is a method that prevents unauthorized users from accessing sensitive information [13]. Authentication is widely used in electronic examinations. Authenticating the students' identity is a very important practice because it helps to reduce academic malpractices and also ensures quality assurance purposes in Education. Both the examinee and examiner should be authenticated in order to secure the E-assessment system. Authenticating the examiner is also an important aspect because the examiner has access to the examinee registration data as well as the examinee assessment data [14].

There are three ways to carry out authentication: (i) knowledge based, which requires a user to provide what he knows (PINS, Usernames, Passwords etc); (ii) object based which requires a user to show what he owns (mobile device, electronic and integrated circuit cards etc); (iii) biometrics (fingerprints, faces etc) $[15,16]$. Most authentication systems make use of the knowledge based and object based methods. This conventional way of authentication for electronic assessment, which is shown in figure 1 , can be altered,compromised,shared or transferred, which can result to impersonation. This poses a potential threat to institutions and administrators of Electronic based examination.

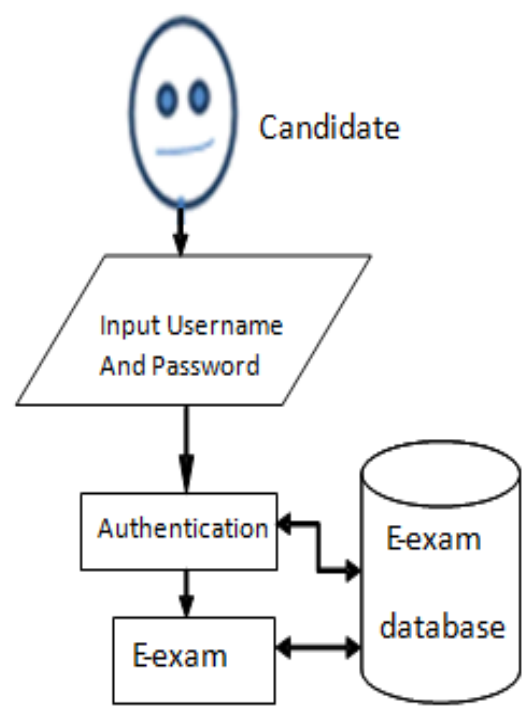

Figure 1: A conventional e-test authentication system (adopted from [12]).

\section{BIOMETRICS-BASED AUTHENTICATION IN E-ASSESSMENT}

The popularity of E-learning in the delivery of educational content and the unprecedented growth of electronic examinations as means of checking learning effect on students has made it necessary to provide a more secure, faster and more suitable method of user's authentication. This can be achieved by the introduction of biometrics technology. Biometrics can be defined as human characteristics and trait related metrics. Biometrics Authentication refers to the identification of humans by their physiological characteristics (such as fingerprint, face, DNA and hand print) and behavioral characteristics (such as signature, gait, voice and typing rhythm). Some of the advantages include universality, distinctiveness, permanence, collectability, performance, acceptability and resistance to circumvention $[12,13]$.

Several research works have been carried out in attempt to eradicate the deficiencies identifies with Electronic examinations. A new approach of using facial recognition for enhanced verification of e-Exam's candidates is presented in [17]. It generates web Application Programming Interface (API) authentication, image, and video with feature extraction matching action. Even though the work attempts to ensure that the candidates who take the Electronic Examination are the right persons, it fails to solve the problems of Face recognition Illumination Variation, Pose/Viewpoint Variation, Facial Expression / Facial Occlusion. The authors of [5] proposed an efficient client/server architecture for face recognition using online webcam in an internet environment. Even though the technique can be applied to confirm users' presence throughout an examination, it requires complex image and video processing to function. The authors of [6] developed an improved e-Learning Authentication and Monitoring System (ELAMS) using logistic Regression (LR) to address the vulnerabilities of e-examination. The system combines Biometric authentication, surveillance (webcam) and machine learning techniques to detect academic dishonesty in an e-learning environment, which improves authentication accuracy but the methods used are complex.

The authors of [18] presented design that uses face recognition for examination identity authentication. The research involves advanced technologies, such as face image capturing, human-machine interaction, data transmission, and communication. The advantages of the system include low cost and high scalability. However, the methods involved in the design are complex.

In [19], the paper attempts to develop an automated face detection and recognition system that can be used for detecting impersonation of candidates in examination system. However, the system fails to handle issues such as face illumination variation. The authors of [7] investigated methods used for cheating detection in online exams, through continuous authentication and online proctors. Their system uses fingerprint reader authenticator and eye tribe tracker in examination session. Even though the research offers some form of continuous authentication, the system is not implemented over the web. In addition, the use of fingerprint for authentication involves making contact with devices which might result to candidates contacting diseases. In [20], 
the authors examined the impacts, challenges and security lapses of the existing electronic-examination system. A new system that uses data encryption for protecting the questions and a biometric fingerprint for authentication was developed. Authentication using fingerprint occurs only at login time, which still gives room for impersonation after the initial login phase. It is also possible for users to contact diseases through the devices. The authors of [21] proposed a continuous authentication system using a multimodal biometric framework. The proposed multimodal framework combines face, mouse dynamics, and keystroke dynamics as the biometric technologies for authentication. The framework has potential for promoting security of electronic examinations, but the reliability of the methods used for the biometrics authentication has not been investigated. The study in [22] examined the existing authentication method for the Computer-based test system of Air Force Institute of Technology (AFIT), Kaduna, Nigeria and proposed the fingerprint biometric technique for authentication. The fingerprint used for authentication does not provide a continuous form of authentication and might result to users contacting diseases. In [12], the research focused on improving security and control by developing a platform based on fingerprint-based technology for authenticating electronic based examination. The platform involves the use mathematical models for fingerprint database, enhancement, feature extraction and pattern matching. Fingerprint technology does not offer continuous authentication and can be a threat to the health of users. The research carried out by [2] addresses the issue of accuracy by proposing an image enhancement approach that uses fingerprint in conjunction with electronic learning environments to eradicate unethical practices with electronic examination in a university environment. It involves extracting minutiae points of fingerprints using the Crossing Number (CN) Concept. The research is limited by the fact that fingerprint technology does not guarantee continuous authentication and is also risky given the fact that users can contact diseases from the devices involved.

\section{PROPOSED BIOMETRIC COMPUTER BASED ASSESSMENT AUTHENTICATION SYSTEM.}

The facial trait is preferred in this research because it can provide a form of continuous authentication. This will solve the problem of impersonation as it ensures that the original candidate is present throughout the examination period [23]. Traits such as fingerprints provide authentication only at login time, which still gives room for impersonation after the initial login. The use of facial trait for authentication is also less expensive, as it doesn't require extra devices for biometric capture. The computer system's camera is used to capture the face, which offers the user a convenient form of authentication. The facial trait also offers a contactless form of authentication in which there is no risk of contacting diseases in an attempt to provide biometric trait. The proposed face biometric system for computer based assessment is shown in Figure 2.It consists of user interface, registration, verification, computer based assessment and database modules. The interface module facilitates visual interaction with the system while the registration module serves as the backbone for pre-registration of examination takers.

\subsection{Verification Module}

The verification module determines the validity of a user in accessing the computer based assessment system. In this phase, the facial trait of a candidate is verified using some sequence of activities.

PCA technique is used to determine eigen faces. For a given $\mathrm{D}$ dimensional data set $\mathrm{X}$ :

First, the mean vector $\mu[\mathrm{d}]$ of dimensions Dx 1 is computed, where $\mathrm{N}$ is the number of samples in the data set:

$$
\mu[d]=\frac{1}{N} \sum_{n=1}^{N} X[d, n]
$$

Compute the DxD covariance matrix $\mathrm{C}$ as follows:

Subtract the mean vector $\mu[\mathrm{d}]$ from each column of the data matrix $\mathrm{X}$ resulting in matrix $\mathrm{B}$ of DxN dimension.

$$
B=X-\mu .1
$$

Compute the covariance matrix $\mathrm{C}$ of $\mathrm{D} \times \mathrm{D}$ dimension:

$$
C=\frac{1}{N} \quad B \cdot B^{*}
$$

The eigenvectors $\left(e_{d}\right)$ and eigenvalues $\left(\lambda_{\mathrm{d}}\right)$ will be extracted as follows:

Eigenvectors: compute matrix $\mathrm{V}$, formed by $\mathrm{D}$ columns vectors of $\mathrm{D}$ length each, which diagonalizes the covariance matrix $\mathrm{C}$.

$$
\begin{aligned}
& V^{-1} C V=\wedge \\
& V=\left[\begin{array}{lll}
e_{1} & \ldots & e_{D}
\end{array}\right]
\end{aligned}
$$

Eigenvalues: the diagonal values of DxD matrix L:

$$
\boldsymbol{\Lambda}=\left[\begin{array}{ccccc}
\lambda_{1} & 0 & \ldots & 0 & 0 \\
0 & \lambda_{2} & \ldots & 0 & 0 \\
\vdots & \vdots & \ddots & \vdots & \vdots \\
0 & 0 & \ldots & \lambda_{\mathrm{D}-1} & 0 \\
0 & 0 & \ldots & 0 & \lambda_{\mathrm{D}}
\end{array}\right]
$$




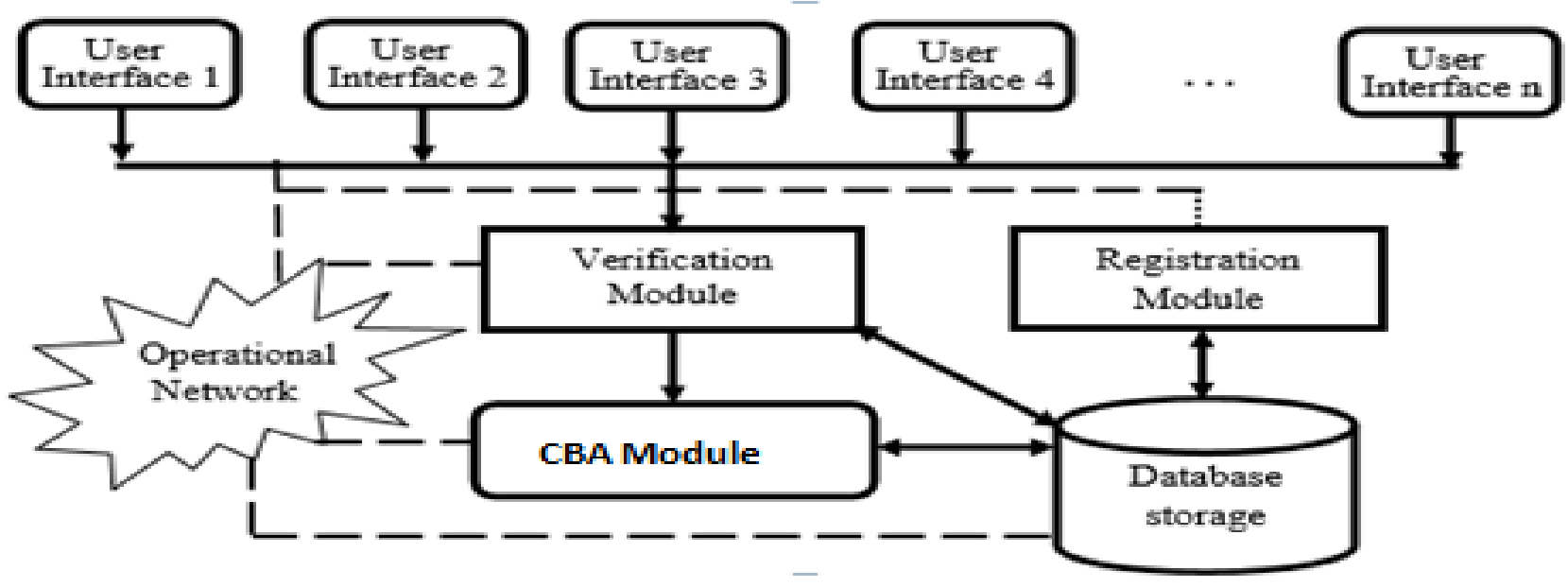

Figure 2: Proposed facial biometric authentication system for Computer Based Assessment (CBA) (adopted from [12]).

The eigenvectors with the largest eigenvalues correspond to the directions of maximum variance. Then, the $\mathrm{L}$ largest eigenvalues and their corresponding eigenvectors have to be chosen, assuming that the rest of the dimension space, D-L, is irrelevant information. Some information will be lost, but the smaller the eigenvalues, the smaller the lost. Once the components (eigenvectors) have been selected, an LxL matrix A with the L eigenvectors as columns has to be formed.

Finally, the transpose of matrix $\mathrm{A}$ has to be taken and multiplied by the original dataset, having previously extracted its mean value.

$$
X^{\prime}=A^{t} \cdot(X-\mu)
$$

The inverse transform to get the original data back can be achieved by applying the following back-projection.

$$
X^{\prime \prime}=A \cdot X^{\prime}+\mu
$$

A facial image can be projected onto $\mathrm{N}^{\prime}$ dimensions by computing

$$
\Omega=\left[\begin{array}{lll}
\mathrm{V}_{1} \mathrm{~V}_{2} & \ldots \mathrm{v}_{\mathrm{N}^{\prime}}
\end{array}\right]^{\mathrm{T}}
$$

Where $v_{i}=e_{d}^{T} B . v_{i}$ is the ith coordinate of the facial image in the new space [24].

The Euclidean distance is minimized to determine which face class provides the good description for input face image as follows:

$$
\mathrm{\epsilon k}=11\left(\Omega-\Omega_{\mathrm{k}}\right) 11
$$

Where $\Omega_{\mathrm{k}}$ is a vector describing the $\mathrm{k}^{\text {th }}$ face class. If ${ }_{\mathrm{Ek}}$ is less than some predefined threshold $\theta_{\mathrm{E}}$, a face is classified as belonging to the class $\mathrm{k}$.

\subsection{Assessment Module}

This is the module used for assessment of candidates that are authenticated successful. This assessment is done through taking of examinations, where candidates are required to provide solutions to the questions been displayed to them. The module computes the result which is used for student assessment. To ensure continuous authentication, the system camera takes the picture of candidates at some specified time intervals. If at any interval the picture taken does not match with the one stored in the database, the system detects an act of impersonation, in which the candidate will be penalized after some warnings. This is done to avoid the situation where a candidate will be impersonated after the initial login.

\subsection{Database Module}

This is the backend for hidden and background operations. It stores information about the candidates, houses the biometric templates and provides querying capabilities.

\section{SYSTEM IMPLEMENTATION}

The system implementation involves the use of Php and MySql which was used for the database design. The environment is characterized by Window 10 operating system on Intel dual Core T6400 processor with 2G RAM and 40GB hard disk. The system was tested by selected students of the department of Mathematics and Computer Science, University of Mkar, Gboko, Nigeria. The system is discussed below.

\subsection{Home page}

This is the first page to be loaded into the browser when its URL (Uniform Resource Locator) is typed into the address bar. The home page can be accessed from any page in the site. The home page provides access to the registration, instructions, and administrator modules. This is shown in figure 3 below. 




Figure 3: Home page

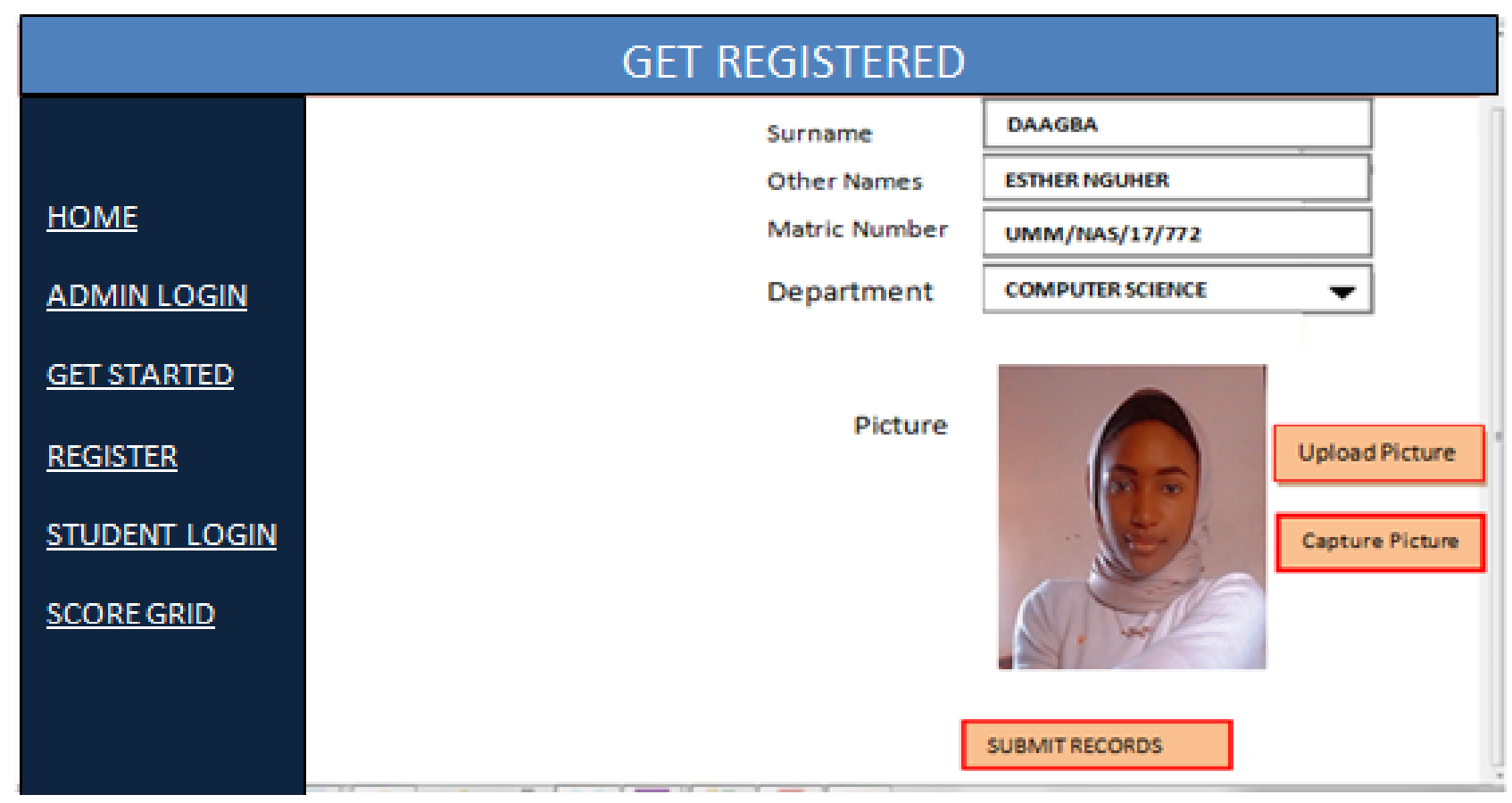

Figure 4: Registration page/Biometric Enrollment.

\subsection{Registration/Biometric Enrollment}

For a user to be eligible for the e-exams, he/she needs to provide details for registration in the registration page as shown in figure 4.

\subsection{Authentication Page}

For a user to be granted access to the exam module, he needs to provide his registration number and face biometric. If the face provided does not match the one stored in the database during registration, authentication fails. If the face biometric provided matches the one stored in the database, the user is authenticated and then he can proceed to write exams. This is shown in figure 5 and figure 6. 


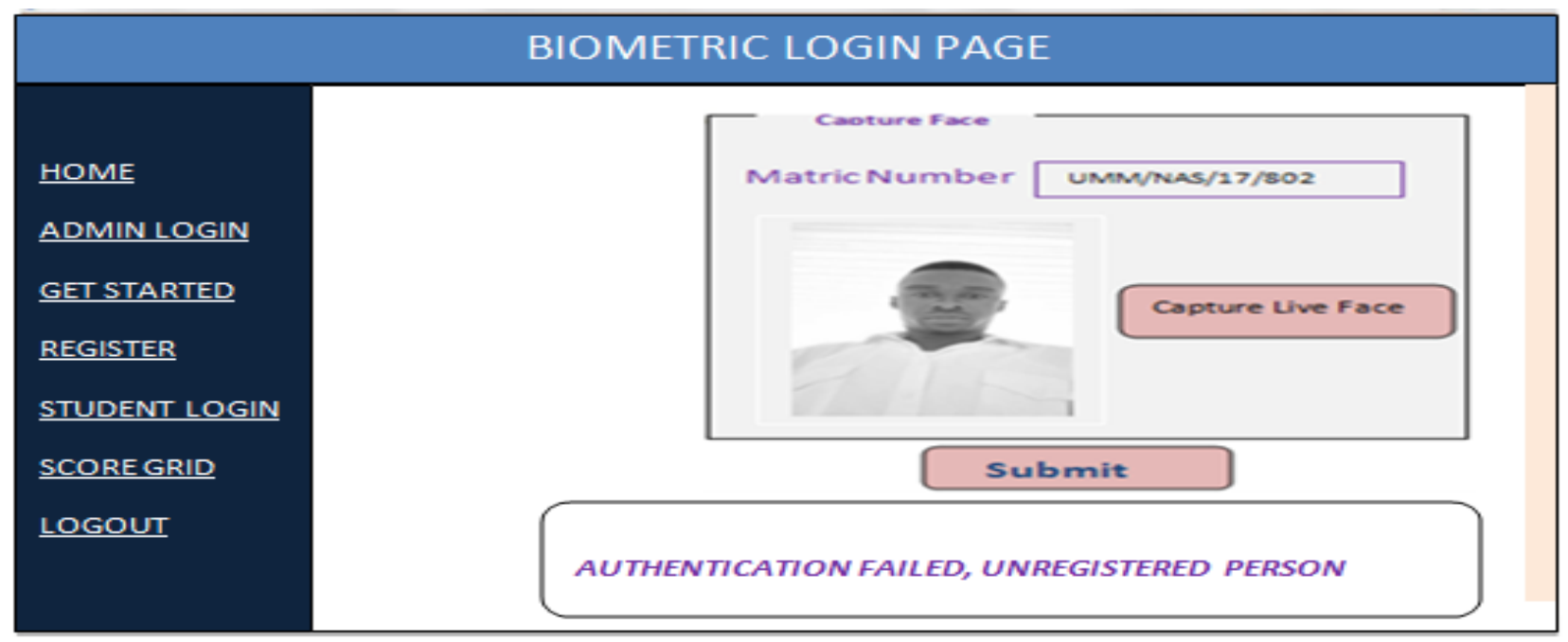

Figure 5: Authentication failure because of wrong biometric

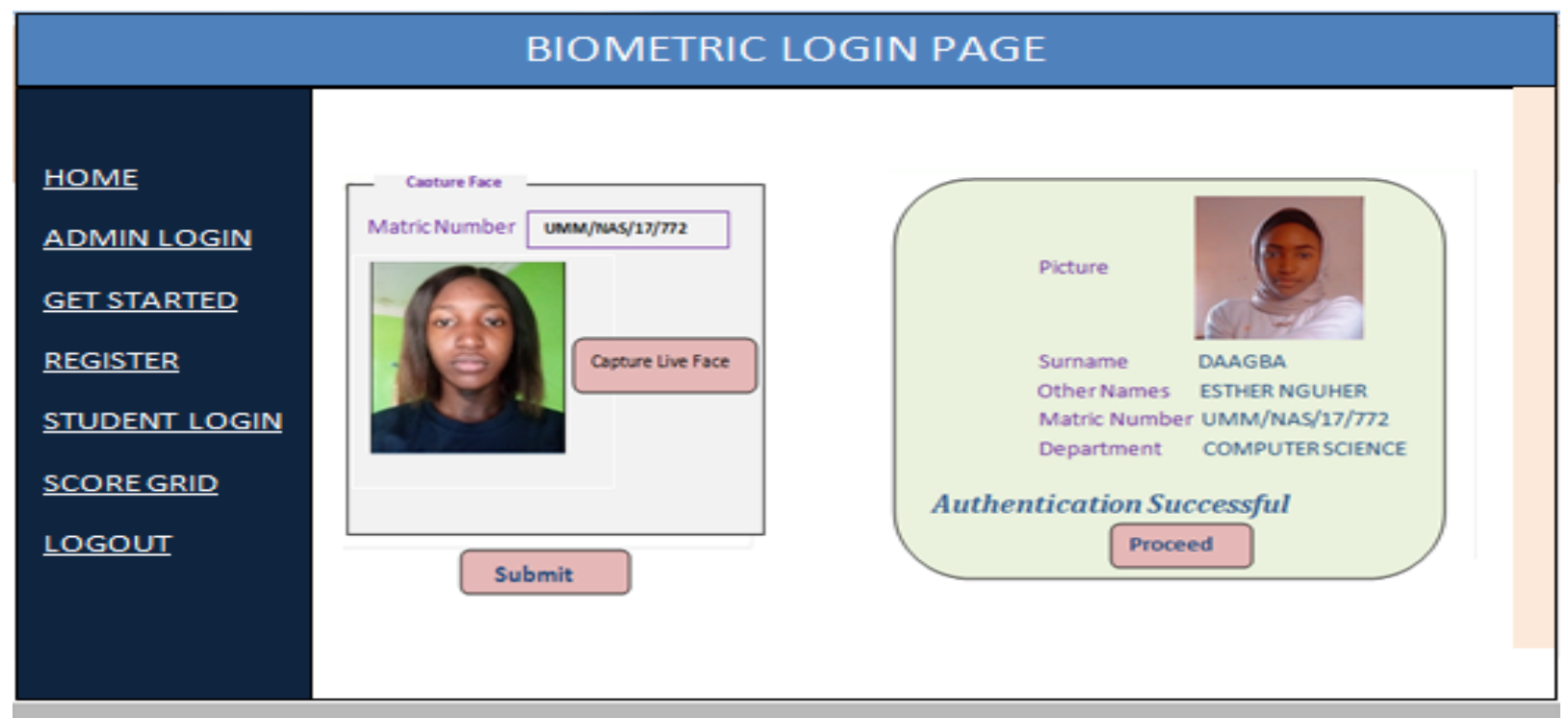

Figure 6: Successful authentication

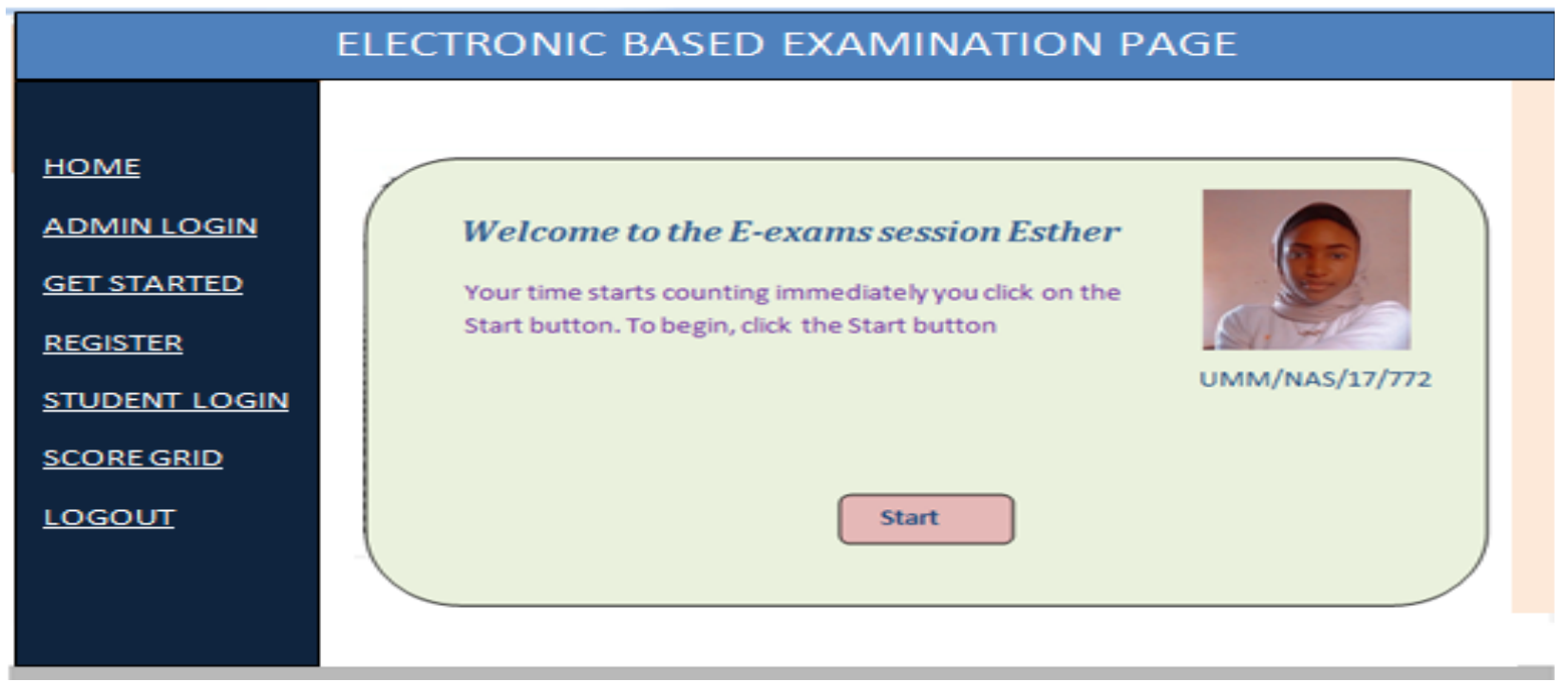

Figure 7: Student assessment page 
Achimba Terfa et al., International Journal of Advanced Trends in Computer Science and Engineering, 11(1), January - February 2022, 26 - 33

\subsection{Assessment Page}

After a successful authentication of the face biometric, the user is then provided with the e-exam page where he/she can now take the examination. This is shown in figure 7.

\section{CONCLUSION}

This research presents a system that makes use of facial biometric traits to authenticate candidates for electronic examinations. Although a face biometric trait is not as accurate as fingerprint, it has a high level of acceptability and provides a form of continuous authentication. With facial biometrics, the system camera captures the candidate's face at different intervals, to make sure that the facial biometric used for the initial login is the face that appears throughout the examination. This will solve the problem of impersonation in electronic examination. The system was tested in the department of Mathematics and Computer Science, University of Mkar, Mkar, Nigeria and the users' acceptability, performances in terms of speed, accuracy, and ease of use were established. Our further research will attempt to make use of Multimodal biometrics for the authentication of examination takers in order to improve on system accuracy.

\section{REFERENCES}

[1] Fayyoumi, A and Zarrad, A (2014). "Novel Solution Based on Face Recognition to Address Identity Theft and Cheating in Online Examination Systems", Advances in Internet of Things, 4, 5-12.

http://dx.doi.org/10.4236/ait.2014.42002.

[2] Mohammed B.I, Abubakar U.O, Olumide S.A and Bukola F.B; "Design of a Fingerprint Biometric Authentication Technique for Electronic Examination", International Journal of Computer Science and Telecommunications [Volume 8, Issue 2, March 2017]

[3] Achimba T (2014). "Design and Implementation of a Computer Based Assessment System”, M.Tech dissertion, Department of Computer Science, Federal University of Technology, Akure.

[4] Alotaibi, S. (2010). "Using Biometrics Authentication via Fingerprint Recognition in E-exams in E-Learning Environment". The 4th Saudi International Conference, Friday 30 and Saturday 31 July 2010, The University of Manchester, UK.

[5] Bruno E.P and Aparecido N.M; "A video-based biometric authentication for elearning web applications", International Conference on Enterprise Information Systems, 2009.

[6] Adetoba, B.T, Awodele, O, Kuyoro S.O, Nwaocha O.V "An Improved Authentication and Monitoring System for E-Learning Examination Using Supervised Machine Learning Algorithms", International Journal of Scientific \& Engineering Research Volume 11, Issue 3, March-2020 ISSN 2229-5518
[7] Razan B, Abdullah B, Anas F and Shehab G; "E-exam Cheating Detection System", International Journal of Advanced Computer Science and Applications, Vol. 8, No. 4, 2017.

[8] Amr J; "New Detection Cheating Method of Online-Exams during COVID-19 Pandemic", International Journal of Computer Science and Network Security, VOL.21 No.4, April 2021

[9] T. Ramu and T. Arivoli, "A framework of secure biometric based online exam authentication: an alternative to traditional exam", International Journal of Scientific \& Engineering Research, Volume 4, Issue 11, November-2013 ISSN 2229-5518.

[10] Mohammad AS and Mohammed I. "Challenges of online exam, performances and problems for online university exam". International Journal of Computer Science Issues. 2013;10(1):439-443.

[11] Peat $\mathrm{M}$ and Franklin S. "Use of online and offline formative and summative assessment opportunities: Have they had any impact on student learning?" Proceeding of ASCILITE; 2002.Available:www.ascilite.org/conferences/au ckland02/proceedings/papers/019.pdf

[12] Iwasokun G. B., Akinyokun O. K., Akinyede R. O and Udoh S. S; "Fingerprint-Based Authorization Platform for Electronic-Based Examination", Journal of Scientific Research \& Reports 12(6): 1-10, 2016; Article no.JSRR.29179 ISSN: 2320-0227.

[13] Achimba T, Alaaga J and Kwaghbee S (2021): "Multi-Modal Biometrics Systems: Concepts, Strengths, Challenges and Solutions", International Journal of Advanced Trends in Computer Science and Engineering, Volume 10, No3 May-June 2021.

[14] Saleh M and Hanif U, "Security Considerations and Recommendations in Computer-Based Testing", The Scientific World Journal Volume 2014, Article ID 562787

[15] Xiaoling $Z$ and Chenglong $C$; "Secure Online Examination with Biometric Authentication and Blockchain-Based Framework", Mathematical Problems in Engineering Volume 2021, Article ID 5058780.

[16] Alexandra O, Denise W, Wayne $\mathrm{H}$ and Chris E. "e-Authentication for online assessment: A mixed-method study", British Journal of Educational Technology, Vol 50, No 2, 2019.

[17] Samson I, Mohd F.R, Maheyzah S and Siti H.O, "A Smart Approach of E-Exam Assessment Method Using Face Recognition to Address Identity Theft and Cheating", International Journal of Computer Science and Information Security (IJCSIS), Vol. 14, No. 10, October 2016.) 
[18] Li-jun Y.U and Ke-feng L.I; "Application of Face Recognition Technology in the Exam Identity Authentication System", 2017 3rd International Conference on Social Science and Management (ICSSM 2017) ISBN: 978-1-60595-445-5

[19] Anuradha.S.G, Kavya.B, Akshatha.S, Kothapalli J and Gudipati A; "Automated Face Detection \& Recognition for Detecting Impersonation of Candidate in Examination System", International Journal of Scientific \& Engineering Research, Volume 7, Issue 3, March-2016 ISSN 2229-5518.

[20] Olawale A and Shafi'i M.A, "E- Exams System for Nigerian Universities with Emphasis on Security and Result Integrity", International Journal of the Computer, the Internet and Management (IJCIM) Volume 18, Number 2: ISSN 0858-7027

[21] Issa T, Youssef N,Sherif S, Bassam S, Julibio D.A, and Paulo M.F.Q; "Ensuring Online Exam Integrity Through Continuous Biometric Authentication", (c) Springer International Publishing AG 2017, Information Security Practices.

[22] Evwiekpaefea, A.E and Eyinlab, V.O; "Implementing Fingerprint Authentication in Computer-Based Tests", Nigerian Journal of Technology Vol. 40, No. 2, 2021, 2021, pp. 284-291

[23] Yousef S, Imane S and Amira K, "Synchronous Authentication with Bimodal Biometrics for e-Assessment: A Theoretical Model",2012 $6^{\text {th }}$ International Conferennce on Sciences of Electronics, Technologies of information and Telecommunications(SETIT), 2012,pp.139-145, doi:10.1109/SETIT.2012.6481902.

[24] Kyungnam Kim, "Face Recognition using Principle Component Analysis", International Conference on Computer Vision and Pattern Recognition, pp.586-591, 1996. 